A few minor errors have also appeared in the text. Thus, the omission of a negative charge from the extruded group $X^{-}$in the bimolecular substitution process on $p .53$, the reference to a diagram as on p. 65 which is really on p. 67 , the possible confusion which may arise (p. 85) by describing $\mathrm{Cl}^{-}$as a chlorine ion instead of the established chloride, the more so as possibly a $\mathrm{Cl}+$ is involved in this reaction, and finally, the reference p. 62 should be to Braude and not Brande.

The book is well and pleasingly produced and adequately indexed. One can confidently look forward to the appearance of a further edition within the near future.

R. I. REED

\section{Fundamentals of Optics}

By Prof. Francis A. Jenkins and Prof. Harvey E. White. Third edition. Pp. vii +637 . (London : McGraw-Hill Publishing Company, Ltd., 1957.) 64s.

TN its third edition, this excellent text-book has 1 been largely rewritten to include recent developments in both experiment and theory, and to bring the treatment into line with modern ideas on the teaching of the subject. There can be few books ranging from the detailed drawing-board computations of ray-tracing, and the analytical discussion of third-and fifth-order aberration, to the scintillation counter and its possible use to examine simple interference experiments in the light of the uncertainty principle. The mathematical proofs of formulae have been presented more concisely, and the complexquantity notation used in the wave-theory section of the book makes for easier reading. The diagrams, many of which illustrate graphically the physical meaning of an analytical expression, are really helpful in supplementing the mathematics. The book is well produced, and can be recommended as very good value.

G. R. NoakeS

\section{Nuclear Magnetic Resonance}

Annals of the New York Academy of Sciences, Vol. 70, Article 4. By H. S. Gutowsky and seventeen other authors. Pp. 763-930. (New York: Now York Academy of Sciences, 1958.)

A CONFERENCE on nuclear magnetic resonance was held by the New York Academy of Sciences in November, 1957, and the papers presented at this meeting have now been published. The papers are concerned with some of the more recent applications of nuclear magnetic resonance to chemical problems. The first part deals with the fundamental interactions which determine the nuclear resonance line-widths and shapes, their chemical shifts and multiplicitios. The origins of these interactions are summarized by C. P. Slichter, and H. S. Gutowsky gives an excellent review of the chemical interpretation of chemical shifts and spin - spin coupling constants and of the principles of the analysis of the sometimes complex high-resolution spectra. This is followed by a description by Schneider, Bernstein and Pople of the detailed analysis of the very complex spectra of pyridine and its deuterium derivatives. The study of hindered internal rotation in organic molecules is discussed by W. D. Phillips, and of chemical exchange kinetics by W. G. Schneider and L. W. Reeves. P. C. Lauterbur describes experiments to explore the use of the spectra obtained from carbon-13 nuclei in natural abundance in organic compounds. The use of high. resolution nuclear resonance spectra to study really complex organic molecules is illustrated by F. C.
Nachod and by M. Saunders and A. Wishnia. The complementary use of nuclear magnetic resonance and infra-red spectra is discussed by $\mathbf{H}$. Finegold with especial reference to the chemistry of organic compounds of phosphorus, and R. B. Williams gives a detailed and authoritative discussion of the conditions necessary for the use of nuclear magnetic resonance for quantitative analysis.

For the reader with some acquaintance with the subject of radio-frequency spectroscopy, this collection of papers provides an excellent survey of the uses of the method in chemistry.

\section{R. E. Richards}

The Preparation of Programs for an Electronic Digital Computer

By Dr. Maurice V. Wilkes, Dr. David J. Wheeler and Dr. Stanley Gill. Second edition. (Addison-Wesley Mathematical Series.) Pp. xiv +238. (Reading, Mass. : Addison-Wesley Publishing Company, Ine.; London: Academic Books, Ltd., 1957.) $60 \mathrm{~s}$.

THIS is a second and enlarged edition of the book first published in 1951. As before, the main emphasis is on the preparation of programmes for Edsac I together with the specification and detailed coding of selected library sub-routines. Additional material has been added, however, on machines possessing different order codes and different input and output systems, while twelve pages are devoted to automatic programming and the concept of 'floating addresses'.

The book is in three parts, its basic plan following that of the first edition. Part 1 gives all the information required to prepare a programme for the Edsac and deals in general terms with other machines. Part 2 specifies the most important of the Edsac library sub-routines and Part 3 gives the complete list of instructions for selected sub-routines.

The book will be of most value to the beginner who wishes to learn some of the principles underlying programming. In particular those intending to programme Edsac I will welcome the revised description of the order code, in which important changes have occurred since the publication of the first edition. These include the provision of a $B$-register, the unconditional jump order and output on to punched paper tape.

The use of the machine is clarified by a number of simple examples, and exercises - many with specimen solutions provided-are set to enable the reader to test his understanding of the machine.

The book has a more attractive layout than previously, and the authors are to be commended on this revised edition.

E. L. Albasiny

\section{Fourier Transforms and $X$-ray Diffraction}

By Prof. H. Lipson and Dr. C. A. Taylor. Pp. vii +76 . (London: G. Bell and Sons, Ltd., 1958.) 18s. 6d. net.

7 Th HIS is a well-prepared book at a comparatively moderate price, suitable for third-year undergraduates and junior postgraduates, but containing much that will interest senior research workers and university teachers. When the theory of X-ray diffraction by crystals is approached by way of the Bragg relation, the transition to the reciprocal lattice can often seem highly artificial, and the idea of the molecular transform may nover be reached at all, especially by the non-mathematically inclined. The authors argue convincingly that if what is interesting is the content of one unit cell, then it is more logical 